\title{
Task-Dependent Presynaptic Inhibition
}

\author{
Marie-Pascale Côté and Jean-Pierre Gossard \\ Centre de recherche en sciences neurologiques, Département de physiologie, Faculté de Médecine, Université de Montréal, Montréal, \\ Québec, Canada, H3C 3J7
}

This study compares the level of presynaptic inhibition during two rhythmic movements in the cat: locomotion and scratch. Dorsal rootlets from L6, L7, or S1 segments were cut, and their proximal stumps were recorded during fictive locomotion occurring spontaneously in decerebrate cats and during fictive scratch induced by D-tubocurarine applied on the $\mathrm{C} 1$ and $\mathrm{C} 2$ segments. Compared with rest, the number of antidromic spikes was increased (by 12\%) during locomotion, whereas it was greatly decreased (31\%) during scratch, and the amplitude of dorsal root potentials (DRPs), evoked by stimulating a muscle nerve, was slightly decreased (7\%) during locomotion but much more so during scratch (53\%). When compared with locomotion, the decrease in the number of antidromic spikes (45\%) and the decrease in DRP amplitude (43\%) during scratch were of similar magnitude. Also, the amplitude of primary afferent depolarization (PAD), recorded with micropipettes in axons $(n=13)$ of two cats, was found to be significantly reduced $(60 \%)$ during scratch compared with rest. During both rhythms, there were cyclic oscillations in dorsal root potential the timing of which was linearly related to the timing of rhythmic activity in tibialis anterior. The amplitude of these oscillations was significantly smaller (34\%) during locomotion compared with scratch. These results suggest that the reduction in antidromic activity during scratch was attributable to a task-dependent decrease in transmission in PAD pathways and not to underlying potential oscillations related to the central pattern generator. It is concluded that presynaptic inhibition and antidromic discharge may have a more important role in the control of locomotion than scratch.

Key words: presynaptic inhibition; fictive locomotion; fictive scratch; dorsal root potential; spinal cord; motor control

\section{Introduction}

The level of sensory feedback required to assist and adjust movements may be controlled by varying levels of presynaptic inhibition in primary afferents. Evidence for such control is primarily based on electrophysiological findings recorded during locomotion in different species (Clarac et al., 1992; Nusbaum et al., 1997), including humans (Morin et al., 1984; Stein and Capaday, 1988; Dietz et al., 1990; Brooke et al., 1991; Yang and Whelan, 1993; Faist et al., 1996). For example, in the cat, there are three types of presynaptic responses associated with stepping that are generated at the level of afferent terminals and conducted back into primary afferent axons. First, there are cyclic oscillations in the membrane potential of a majority of afferents accompanying the rhythm of the central pattern generator (CPG) for locomotion. These CPG-related oscillations may be recorded at the level of cut dorsal rootlets (Baev, 1980; Baev and Kostyuk, 1982; Dubuc et al., 1985, 1988), single primary afferents (Gossard et al., 1989,1991 ), or as excitability changes of afferent terminals (Baev, 1980; Baev and Kostyuk, 1982; Duenas and Rudomin, 1988). Second, antidromic discharges may occur as rhythmic bursts that are synchronized with the step cycle, as seen in cut dorsal rootlets (Dubuc et al., 1985, 1988; Beloozerova and Rossignol, 1994, 1999; Rossignol et al., 1998) or in single cutaneous (Gossard et al., 1989) and muscle (Gossard et al., 1991) afferents. Third, there is

\footnotetext{
Received June 3, 2002; revised Dec. 9, 2002; accepted Dec. 9, 2002.

This work was supported by a grant from the Canadian Institutes of Health Research. M.-P.C. was supported by the joint Fonds pour la Formation de Chercheurs et l'aide à la Recherche du Québec and Fonds de la Recherche en Santé du Québec. We thank F. Lebel for technical support.

Correspondence should be addressed to Dr. Jean-Pierre Gossard, Centre de recherche en sciences neurologiques, Département de physiologie, Faculté de Médecine, Université de Montréal , C.P. 6128, Succ. Centre-ville, Montréal, Québec, Canada, H3C 3J7. E-mail: jean-pierre.gossard@umontreal.ca.

Copyright $\odot 2003$ Society for Neuroscience $\quad$ 0270-6474/03/231886-08 $\$ 15.00 / 0$
}

a phase-dependent modulation of the amplitude of primary afferent depolarization (PAD) evoked by diverse sensory stimulation during fictive stepping as recorded in individual cutaneous (Gossard et al., 1990) and muscle (Ménard et al., 1999) afferents or as dorsal root potential (DRP) (Gossard and Rossignol, 1990). This phase-dependent modulation is a strong indication that the excitability of PAD interneurons is under the control of the CPG for locomotion.

In several other motor tasks, the presence of presynaptic inhibition was either recorded or proposed to explain changes in reflexes. For example, there is one report showing cyclic oscillations and antidromic firing in dorsal rootlets during fictive scratch in decerebrated cats (Baev and Kostyuk, 1981). Different frequencies of antidromic firing were recorded in the central axons and the soma of trigeminal muscle spindle afferents during fictive mastication in rabbits, suggesting a CPG-related increase in excitability of central axons (Westberg et al., 2000). Cyclic oscillations in afferent membrane potential were reported during fictive respiration in the cat (Richter et al., 1986) and the crab (DiCaprio, 1999) and during fictive mastication in the guinea pig (Kurasawa et al., 1988). Is presynaptic activity specifically programmed for each task or is it just turned on whenever motor pools are recruited? If presynaptic inhibition were to play a role in gating sensory feedback, then one would expect each motor task to have a specific program of presynaptic activity. We investigated this issue by comparing presynaptic responses during fictive locomotion and scratch in decerebrate cats. Some of the results have been published previously in abstract form (Côté and Gossard, 2000, 2001).

\section{Materials and Methods}

All procedures were conducted according to the Guide for Care and Use of Experimental Animals, using protocols approved by the Ethics Committee of the University of Montreal (Montreal, Canada). 
Acute experiment. Nine female cats $(2.7-4.2 \mathrm{~kg})$ were used for this study. Cats were first anesthetized by inhalation of an oxygenated mixture $(50 \%)$ of nitrous oxide $(50 \%)$ and halothane $(2-3 \%)$ (MTC Pharmaceuticals, Cambridge, Ontario, Canada). Cannulas were inserted in the right common carotid artery to monitor blood pressure and in jugular and cephalic veins for administration of pharmacological agents or fluids.

The following muscle and cutaneous nerves from the left hindlimb were dissected free, cut, and mounted on bipolar silver chloride electrodes for recording [electroneurogram (ENG)] and stimulation: posterior biceps-semitendinosus (PBSt), semimembranosus-anterior biceps $(\mathrm{SmAB})$, lateral gastrocnemius-soleus (LGS), medial gastrocnemius (MG), plantaris, flexor hallucis longus and flexor digitorum longus (FDHL), tibialis anterior (TA), superficial peroneal (SP, uncut), caudal cutaneous sural, and the sciatic nerve (Sci, uncut).

A laminectomy (L4-L7) was then performed, and the animal was transferred to a stereotaxic frame. After an extensive craniotomy, the animal was decerebrated by making a precollicular-postmammillary transection (cf. Gossard, 1996; Ménard et al., 1999), and anesthesia was discontinued after signs of decerebrate rigidity. The animal was then paralyzed (Pavulon, $0.2 \mathrm{mg} / \mathrm{kg}, 45 \mathrm{~min}$; Sabex, Boucherville, Ontario, Canada) and artificially ventilated, maintaining the $\mathrm{CO}_{2}$ level near $4 \%$. Pools were constructed with skin flaps surrounding the exposed spinal cord and the hindlimb nerves and filled with warm mineral oil. Body and pool temperature was kept near $38^{\circ} \mathrm{C}$ with an infrared lamp. A second laminectomy exposing $\mathrm{C} 1$ and $\mathrm{C} 2$ segments was also performed to allow topical application of D-tubocurarine to induce scratch.

Stimulation, recordings, and analysis. The cord dorsum potential (CDP) was recorded with a silver chloride ball electrode located near the dorsal root entrance at the L6-L7 border. Stimulation intensity required to evoke just a deflection in the CDP determined the threshold for the most excitable fibers for each nerve $(1 \mathrm{~T})$. Stimulus intensity will be expressed as a multiple of the threshold.

Dorsal rootlets (L6, L7, S1) were cut, and proximal stumps were dissected in thin filaments, which were recorded with bipolar silver chloride electrodes (Dubuc et al., 1988; Gossard and Rossignol, 1990; Gossard et al., 1999). In two experiments, intra-axonal recordings of identified primary afferents in dorsal columns (Gossard et al., 1989, 1991; Ménard et al., 1999) were performed (L6-S1) with glass micropipettes filled with $\mathrm{K}^{+}$-acetate $(2 \mathrm{M})$ and $N$-(2,6-dimethylphenylcarbamoylmethyl) triethylammonium chloride (QX314) (100 mM; Alamone Laboratories, Jerusalem, Israel) to prevent sodium spikes. Identification of primary afferents (Gossard et al., 1989, 1991; Ménard et al., 1999) included the threshold for activation $(<2.0 \mathrm{~T}$ for group I; $2.0-5.0 \mathrm{~T}$ for group II); the ability to follow electrical stimulation of a specific skin, muscle nerve, and/or sciatic nerve at high frequency $(>500 \mathrm{~Hz})$ with a short and constant latency and the absence of a prepotential on the evoked spike; and their responses to cutaneous stimuli or muscle stretches.

Activity in muscle nerves, dorsal rootlets, and axons was amplified and recorded. All signals were recorded on videotape after digitalization (15 channels; Vetter 4000A; A.R. Vetter, Rebersburg, PA). Tapes were played back off-line on an electrostatic printer (Gould ES-1000; Gould Instrument, Valley View, OH), and portions of interest were digitized and analyzed with interactive custom-made software (Spinal Cord Research Center, University of Manitoba, Winnipeg, Canada).

A small cotton ball soaked in D-tubocurarine $(0.1 \%$; Sigma, St. Louis, MO) was applied topically at C1 and C2 levels (Feldberg and Fleischhauer, 1960), and episodes of fictive scratch either were occurring spontaneously or were induced by manual stimulation of the left pinna (Deliagina et al., 1975, 1981). Episodes of fictive locomotion occurred spontaneously. The two rhythms were easily distinguished by their different pace, with scratching being three to four times faster than fictive stepping (see Fig. 2). Also, the extensor activity occupies only $20 \%$ of the fictive scratch, whereas during fictive locomotion, it occupies a much larger proportion of the cycle (Deliagina et al., 1975; Kuhta and Smith, 1990). Only episodes with vigorous and regular ENG bursts during locomotion or scratch were selected for analysis. Also, continuous bouts of ENG signals with a complete absence of bursts were selected as rest episodes.

The total number of antidromic spikes in a given rootlet was calculated and compared for episodes of scratching, stepping, and rest for an episode of same duration. Locomotor or scratch cycle is defined as the period between the onsets of two successive bursts of activity in the ankle flexor nerve TA. The flexor phase was determined by the duration of TA burst, whereas the extensor phase was determined by the duration of LGS or MG bursts. Because cycles vary in length, they were normalized to the same length so that both the start and the end of the cycle line up. To compare the spontaneously occurring potential excursion of dorsal rootlets in scratch and locomotion (CPG-related oscillations), the dorsal rootlets signals were averaged for several cycles. Dorsal root potentials were evoked by stimulating PBSt (three pulses, 2 T, $300 \mathrm{~Hz}$ ). Primary afferent depolarization was evoked by stimulating PBSt (three pulses, $2 \mathrm{ST}, 5 \mathrm{~T}, 300 \mathrm{~Hz}$ ). DRPs and PADs were evoked every $300 \mathrm{msec}$, a frequency of stimulation that is fast enough to obtain an optimal number of responses during rhythmic episodes that are limited in time but slow enough so that responses do not influence each other. The peak amplitude of DRPs and PADs was measured off-line and averaged for episodes of rest, scratch, and locomotion.

To assess the temporal relationship between ENG bursts (onsets and offsets) and CPG-related oscillations, the time duration, from the beginning of the cycle to the end of the depolarization in CPG-related oscillations in dorsal rootlet potential (see $x$ in Fig. 4A) and the TA burst duration (see $x^{\prime}$ in Fig. $4 A$ ) were measured; these two values were then analyzed with linear regression.

Statistical analysis. Statistical analysis was performed to disclose differences in the number of antidromic spikes, the timing and amplitude of CPG-related oscillations, DRP, and PAD amplitude obtained during rest, locomotion, and scratch. Measurements were compared within the same rootlet or axon. Values obtained in locomotion or scratch were also expressed as percentages of values obtained at rest (Table 1, columns 4 and 6; Table 2, columns 4 and 7), and values obtained in scratch were expressed as percentages of values obtained in locomotion (Table 1, column 7; Table 2, column 9). Statistical analysis was also applied to disclose differences between these percentages. The Kolmogorov-Smirnov-Liliefors (KSL) test was used to compare the shape and location of the distribution of responses with a normal distribution. If the KSL test confirmed that the sample variables did fit a normal distribution, the parametric Student's $t$ test was performed; if not, the nonparametric Mann-Whitney rank sum test was used.

\section{Results}

In all cats $(n=9)$, we could compare presynaptic responses at rest and during fictive scratch and, in five cats, during fictive locomotion as well. Responses were collected from one to three rootlets (L6, L7, S1). In the first part, presynaptic potentials and firing, occurring spontaneously during rhythm generation, were recorded at the level of dorsal rootlets (Fig. $1 A$ ). In the second part, responses mediated by presynaptic inhibitory pathways were evoked by stimulating a peripheral muscle nerve (Fig. $1 B$ ). The evoked responses were recorded at the level of dorsal rootlets and intracellularly in individual primary afferents.

\section{Antidromic discharges}

Figure 2 illustrates $10 \mathrm{sec}$ of activities in dorsal rootlets recorded during three episodes that occurred within $2 \mathrm{~min}$. The episode of spontaneous fictive locomotion was followed by an episode of rest, where there was an absence of bursts in ENGs, which was followed by an episode of fictive scratch evoked by gentle pinna stimulation. During both stepping and scratching, the potential of the two rootlets (L7 and S1) was oscillating in time with the rhythmic bursts of activity in the muscle nerves. Also, numerous spikes of different amplitude were seen in the two cut rootlets. Moreover, more firing occurred during the depolarized phase of the rootlets during fictive stepping (Dubuc et al., 1985, 1988; Gossard et al., 1991). The total number of antidromic spikes was compared for rest and fictive rhythms for a similar duration 
Table 1. Total number of antidromic action potentials in dorsal rootlets (DR) during episodes of rest, fictive locomotion (Loco) and scratch of same duration

\begin{tabular}{|c|c|c|c|c|c|c|}
\hline$\overline{\mathrm{DR}}$ & Rest & Loco & (\%Rest) & Scratch & (\%Rest) & Scr $(\% \mid l c)$ \\
\hline L6 & 294 & & & 267 & 90.8 & \\
\hline L7 & 227 & & & 134 & 59.0 & \\
\hline L6 & 586 & & & 397 & 67.7 & \\
\hline L7 & 200 & & & 194 & 97.0 & \\
\hline S1 & 294 & & & 358 & 121.8 & \\
\hline L7 & 120 & 109 & 90.8 & 23 & 19.2 & 21.1 \\
\hline S1 & 144 & 219 & 152.1 & 105 & 72.9 & 47.9 \\
\hline L7 & 235 & 224 & 95.3 & 102 & 43.4 & 45.5 \\
\hline S1 & 302 & 171 & 56.6 & 109 & 36.1 & 63.7 \\
\hline L7 & 169 & 172 & 101.8 & 149 & 88.2 & 86.6 \\
\hline S1 & 563 & 1000 & 177.6 & 263 & 46.7 & 26.3 \\
\hline L6 & 319 & 421 & 132.0 & 303 & 95.0 & 72.0 \\
\hline L7 & 309 & 187 & 60.5 & 146 & 47.2 & 78.1 \\
\hline L6 & 273 & 503 & 184.2 & 321 & 117.6 & 63.8 \\
\hline \multirow[t]{2}{*}{ L7 } & 779 & 568 & 72.9 & 269 & 34.5 & 47.4 \\
\hline & & & 112.4 & & 69.1 & 55.2 \\
\hline
\end{tabular}

Spikes were counted from cut dorsal rootlets from lumbar segments $\mathrm{L} 6, \mathrm{~L} 7$, or $\mathrm{S} 1$. In the fourth and sixth columns, values are expressed as percentages of the number of antidromic spikes recorded during an episode of rest (\%Rest). In the seventh column, values obtained in scratch as percentages of the number of spikes recorded during locomotion are shown [scr(\%loc)]. Numbers in bold at the bottom of columns 4,6 , and 7 are the averaged percentages.

Table 2. Averaged amplitude of DRPs (in microvolts) evoked by muscle afferent stimuli during episodes of rest, fictive locomotion, and scratch

\begin{tabular}{|c|c|c|c|c|c|c|c|c|c|}
\hline$\overline{\mathrm{DR}}$ & Rest & Loco & (\%Rest) & $p<$ & Scratch & (\%Rest) & $p<$ & Scra(\%loc) & $p<$ \\
\hline L6 & 12.97 & & & & 5.72 & 44.1 & 0.001 & & \\
\hline L7 & 18.38 & & & & 15.10 & 82.2 & 0.160 & & \\
\hline L6 & 9.73 & & & & 2.54 & 26.1 & 0.001 & & \\
\hline L7 & 6.11 & & & & 1.88 & 30.8 & 0.001 & & \\
\hline L7 & 4.88 & 5.35 & 109.6 & 0.824 & 3.34 & 68.4 & 0.021 & 62.4 & 0.005 \\
\hline S1 & 6.78 & 5.34 & 78.8 & 0.069 & 3.12 & 46.0 & 0.001 & 58.4 & 0.003 \\
\hline L7 & 9.55 & 7.46 & 78.1 & 0.001 & 3.52 & 36.9 & 0.001 & 47.2 & 0.001 \\
\hline S1 & 6.08 & 4.38 & 72.0 & 0.001 & 3.27 & 53.8 & 0.001 & 74.7 & 0.001 \\
\hline L6 & 7.13 & 6.95 & 97.5 & 0.115 & 4.11 & 57.6 & 0.001 & 59.1 & 0.001 \\
\hline L7 & 5.70 & 5.4 & 94.7 & 0.001 & 2.52 & 44.2 & 0.001 & 46.7 & 0.001 \\
\hline L6 & 17.69 & 24.47 & 138.3 & 0.001 & 7.32 & 41.4 & 0.001 & 29.9 & 0.001 \\
\hline \multirow[t]{2}{*}{ L7 } & 12.76 & 10.59 & 83.0 & 0.025 & 8.59 & 67.3 & 0.001 & 81.1 & 0.001 \\
\hline & & & 93.2 & & & 47.0 & & 57.4 & \\
\hline
\end{tabular}

Abbreviations as in Table 1. Only significant values are used for averaging (numbers in bold at the bottom of columns 4 and 7). Columns 5, 8, and 10 show the level of significance.

$(8-30 \mathrm{sec})$. The results from a total of 15 rootlets in nine cats are compiled in Table 1. Overall, there was a significant difference $(p<0.01)$ between locomotion and scratch in the number of antidromic spikes relative to rest (Table 1, columns 4 and 6 ). Compared with rest, fictive stepping increased the number of antidromic spikes in 5 of 10 cases (average, by 12\%) whereas fictive scratching decreased that number in 13 of 15 cases (average, by $31 \%$ ). When compared with locomotion, there was $45 \%$ less antidromic activity during scratching overall. We then investigated whether differences in the timing or amplitude of CPGrelated oscillations in rootlets (Figs. 3 and 4) or differences in transmission of presynaptic inhibitory pathways (Figs. 5 and 6) could explain such a decrease in antidromic traffic.

\section{CPG-related oscillations in dorsal root potential}

The potential excursion of the two rootlets (CPG-related oscillations) shown in Figure 2 was averaged according to the step cycles $(n=51)$ and scratch cycles $(n=41)$ in Figure 3. Also shown are the rectified and averaged activities in ankle flexor (TA) and extensor (LGS) nerves. If antidromic spikes are generated when the underlying CPG-related oscillations reach firing threshold (Dubuc et al., 1988; Gossard et al., 1991) then, based on the preceding result, one would expect larger depolarization in rootlets during stepping than scratching. A simple visual examination of Figure 3 indicates that the amplitude of CPG-related oscillations in rootlets is actually smaller during fictive locomotion. This issue was further analyzed during both rhythms with statistical analysis (see below). Also, from Figure 3, the patterns of CPG-related oscillations appear to be different during locomotion and scratch. During stepping, the potential of L7 and S1 rootlets follows more or less the same pattern, reaching a maximum depolarization just before the middle of the cycle. The two rootlets also follow a similar pattern during scratch; however, the maximum depolarization is reached toward the end of the cycle. This apparent disparity is actually attributable to the different phasic structure of the cycles. As reported previously (Deliagina et al., 1975; Kuhta and Smith, 1990), the period of activity in flexors is a much larger part of the scratching cycle than it is for the stepping cycle. With that in mind, one can see in Figure 3 that the peak of depolarization in the rootlets occurs toward the end of the flexor phase illustrated by the bursts in TA during both rhythms. We thus also analyzed further the timing of the CPG-related oscillations during both motor activities.

The comparison of the averaged peak-to-peak amplitude (Fig. $4 A, A M P)$ of CPG-related oscillations in the $\mathrm{L} 7$ and $\mathrm{S} 1$ rootlets (Fig. 2) during fictive locomotion and scratch showed that it was significantly smaller $(p<0.001)$ during stepping than scratching in both rootlets. Overall, comparison of 12 rootlets in six cats showed that CPG-related oscillations were 34\% smaller during stepping than scratching (at least $p<0.01$ in 9 of 12 rootlets). We then evaluated the synchronicity between CPG-related oscillations and rhythmic ENG activities. Specifically, the link between 
A

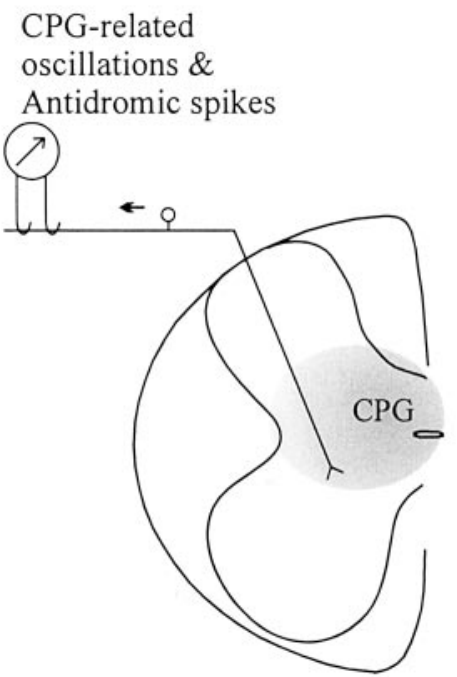

B

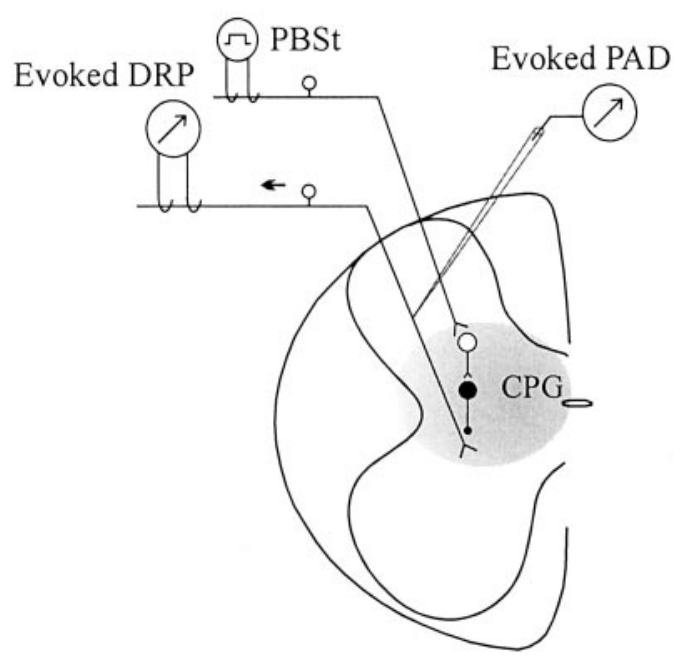

Figure 1. Schematic representation of experimental setup. A, Spontaneous rhythmic oscillations of potential (CPG-related oscillations) and antidromic discharges are recorded in cut dorsal rootlets at L6, L7, or S1 levels during rest and fictive locomotion and scratch. B, The stimulation of the muscle nerve PBSt evokes, through a short chain of interneurons, a primary afferent depolarization in terminals, which may be recorded in a population of axons in dorsal rootlets (dorsal root potential) or in a single axon with a micropipette.

Fictive locomotion

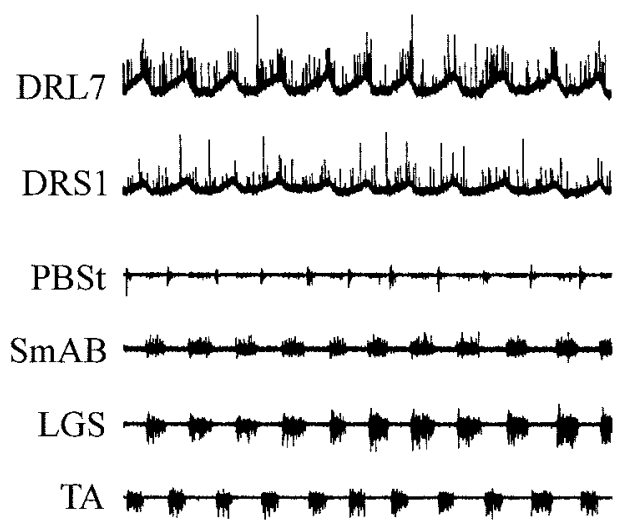

Rest
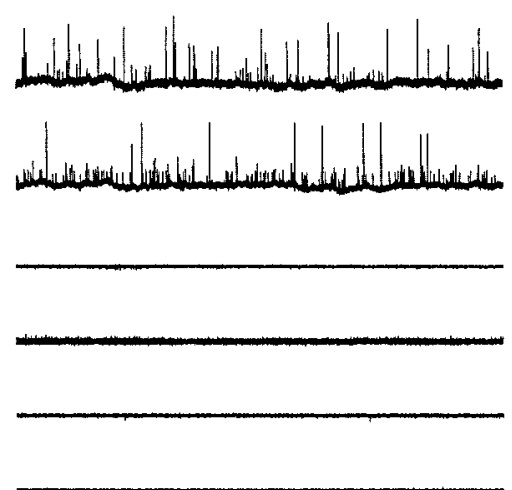

Fictive scratching
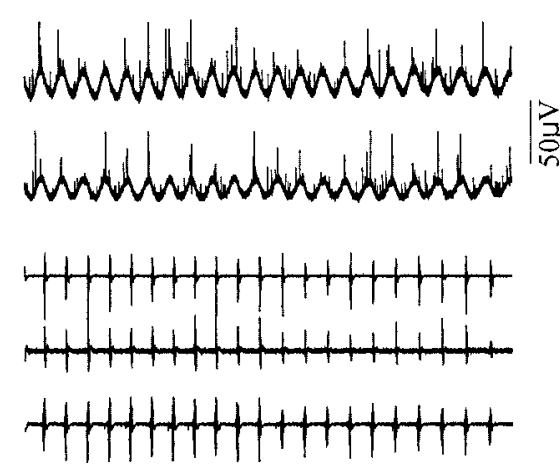

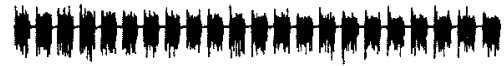

Figure 2. Dorsal root activity during fictive locomotion (left), rest (middle), and fictive scratching (right). Raw recordings of two cut dorsal rootlets and of muscle nerves during three episodes occurring within 2 min are shown. Top to bottom, Dorsal rootlet from L7 and S1 levels (DRL7 and DRS1) and ENGs of PBSt, SmAB, LGS, and TA.

the time of the beginning of the cycle to the end of the depolarization in the dorsal rootlet (Fig. $4 A, x$ ) and the duration of bursts of activity in TA (Fig. $4 A, x^{\prime}$ ) was analyzed with linear regression. In Figure $4 B$, the top two panels are taken from an L7 rootlet during stepping and scratching, respectively, whereas the bottom two panels are taken from the S1 rootlet (Fig. 2). The linear relationships were highly significant $(p<0.001)$ during both stepping and scratching. Overall, significant relationships $(p<0.05)$ were found between these two events in 12 of 12 rootlets during both rhythms. Thus, as estimated by the last measurements, the timing of CPG-related oscillations of polarization is tightly related to the timing of motor activities in both rhythms, but their amplitude is smaller during locomotion.

\section{Sensory-evoked DRPs and PADs}

Next, we investigated whether differences in antidromic traffic could be caused by differences in transmission of the presynaptic inhibitory pathways as activated by sensory feedback. Figure 5 shows the averaged DRP $(n=47)$ evoked by PBSt stimuli at rest (black), during fictive stepping (dark gray), and during fictive scratching (light gray) in L7 and S1 rootlets from one cat. It is clear that the DRP amplitude in both rootlets is dramatically decreased during scratch. During stepping, there is a slight decrease in the S1 rootlet compared with rest. The amplitude of DRPs ( $n=33-149)$ evoked by the same stimuli was measured and averaged from a total of 12 rootlets in six cats, and the results are compiled in Table 2. Compared with rest, the DRP amplitude was significantly decreased in six of eight rootlets $(p<0.01$ in four rootlets) during stepping (average, by $7 \%$ ), whereas it was much more decreased in 12 of 12 rootlets $(p<0.001$ in 10 rootlets) during scratching (average, by 53\%). Overall, there was a significant difference between locomotion and scratch in the DRP amplitude relative to rest $(p<0.001)$ (Table 2, columns 4 and 7). When compared with stepping $(n=8)$, the DRP amplitude was reduced by $43 \%$ during scratching. 


\section{Locomotor cycle}

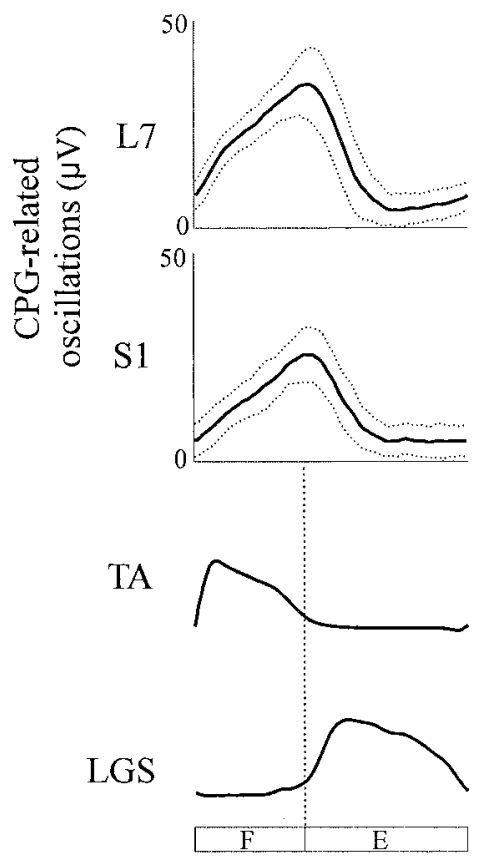

Scratch cycle

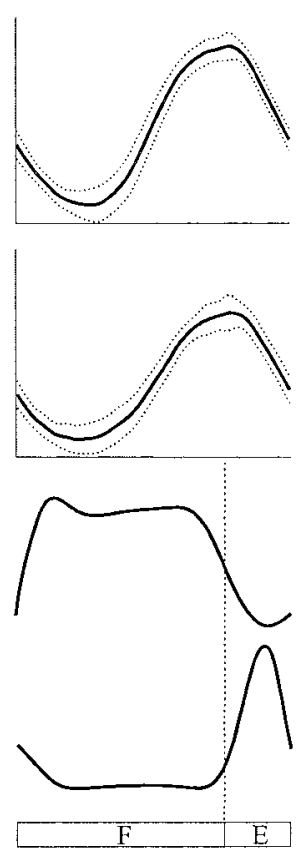

Figure 3. Averaged CPG-related oscillations in dorsal rootlets L7 and S1 (with SD, dotted lines) relative to the step (left) and the scratch cycle (right) together with the averaged rectified activities in TA and LGS taken from Figure 2. F, Flexion phase; $E$, extension phase.

Finally, we thought the rapid oscillations in dorsal rootlets during scratch could have resulted in an underestimation of the amplitude of evoked DRPs. We thus verified whether there was indeed a decrease in presynaptic inhibitory responses during scratch by measuring PAD in axons of primary afferents of the hindlimb, where CPG-related oscillations are quite smaller than in dorsal rootlets (Gossard et al., 1989, 1991). Figure 6 shows the averaged PAD and DRPs (L6 and L7) evoked by PBSt stimuli at rest $(n=48)$ and during fictive scratch $(n=87)$. In both the axon and dorsal rootlets, the responses are dramatically decreased during scratch. This spectacular decrease lasted the entire duration of the scratching episode, as illustrated in Figure $6 \mathrm{~B}$. The graph depicts the amplitude of each PAD evoked during episodes of rest, scratch, and then rest again. It is clear that, at the moment scratching activity began, there was a drop in PAD amplitude. It is interesting to note that the PAD amplitude did not resume toward previous values for several seconds afterward. We recorded 13 axons; of these, 11 were of large caliber [ 5 muscle group I (1 LGS, 1 SmAB, and 3 FDHL), 1 cutaneous SP, and 5 Sci] and 2 Sci axons were of group II or $\mathrm{A} \beta$ caliber. Overall, compared with rest, $\mathrm{PAD}$ amplitude was significantly $(p<0.05)$ decreased during scratch in 11 of 13 axons in two cats (average, by 60\%). There was no relationship between the degree of reduction and the identity of the axons.

\section{Discussion}

The comparison of fictive locomotion and fictive scratch has revealed significant differences in presynaptic potentials and activity. First, there was a dramatic decrease (by 45\%) in the number of antidromic spikes during scratch compared with locomotion. Note that we compared episodes of the two tasks (and of rest) of same duration as a global estimate of antidromic activity. We also investigated whether reasons for such a decrease could be found in the other presynaptic responses occurring during the two tasks. At first, we looked at the timing and the amplitude of CPG-

A

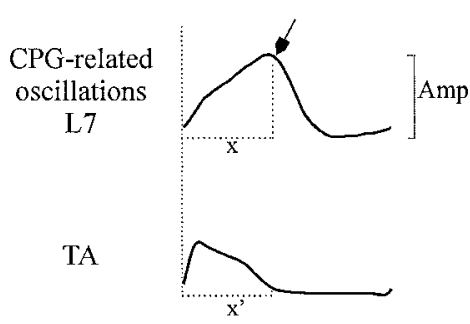

B
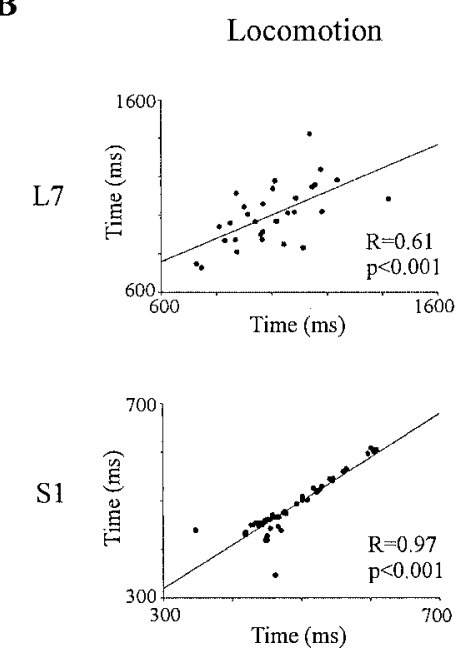

Locomotion
Scratching

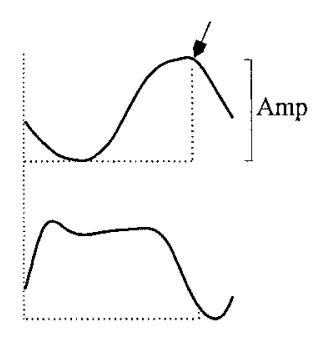

Figure 4. The amplitude and timing of CPG-related oscillations during fictive locomotion and scratch. $A$, The peak-to-peak amplitude (Amp) of potential excursions in dorsal rootlets was measured for each cycle and compared for both rhythms. The time when the end of the maximal depolarization phase in dorsal rootlets occurs $(x)$ and the duration of the TA burst of activity $\left(x^{\prime}\right)$ are related, with linear regression shown in $B$. $B$, Linear relationship between the end of depolarization in L7 and S1 rootlets and the duration of TA activity during locomotion and scratch.

related oscillations in rootlet potential accompanying each task. These oscillations were often considered to be the cause of antidromic firing when they reach firing threshold (Dubuc et al., 1985, 1988; Gossard et al., 1991; Cattaert et al., 1994). There was a significant linear relationship between the timing of potential oscillations (the end of depolarization) and the end of the flexor burst in TA during both tasks. However, results from Baev and Kostyuk (1981) suggested that the maximal depolarization occurred at the beginning of the extensor activity instead. The reasons for this timing difference are unknown. Nevertheless, a tight synchronization between CPG-related oscillations in dorsal rootlets and motor activities was reported both during scratch (Baev and Kostyuk, 1981) and during locomotion (Baev and Kostyuk, 1982; Dubuc et al., 1988). However, the peak-to-peak amplitude of oscillations was found to be $34 \%$ smaller during locomotion compared with scratch. Therefore, it would be difficult to explain the decrease in antidromic firing during scratch as being a result of differences in the underlying potential oscillations; their timing is similar and their amplitude is actually larger compared with locomotion.

Second, we also investigated whether the decrease in antidromic firing was related to differences in transmission in presynaptic inhibitory pathways. These are classically activated in response to sensory or supraspinal inputs (Eccles, 1964; Schmidt, 


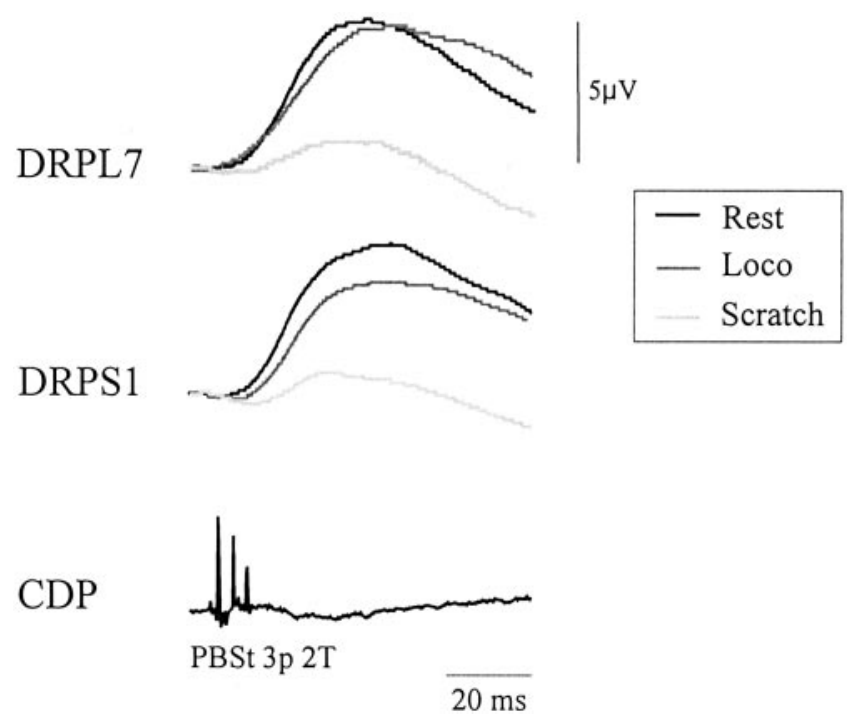

Figure 5. Sensory-evoked DRPs during rest, fictive locomotion (Loco), and scratch. Averaged DRPs in an L7 (DRPL7) and S1 (DRPS1) rootlet evoked by PBSt stimuli at rest (black) and during fictive locomotion (gray) and scratch (light gray) are superimposed. Voltage scale applies to all DRPs. The CDP shows incoming volleys.

1971; Nicoll and Alger, 1979; Rudomin and Schmidt, 1999). Lastorder interneurons are GABAergic and capable of decreasing transmitter release through axo-axonic contacts on terminals evoking a primary afferent depolarization lasting $\sim 100 \mathrm{msec}$ (Eccles et al., 1961; Eccles, 1964; Schmidt, 1971; Nicoll and Alger, 1979). In this study, we stimulated PBSt, which is an efficient sensory source frequently used for studies of presynaptic inhibition in the cat (Ménard et al., 1999; Rudomin and Schmidt, 1999). There was a significant decrease (by 43\%) in the amplitude of DRPs during scratch compared with locomotion. PADs were also recorded in single primary afferents with intra-axonal recordings during scratch (for the first time) and at rest. It was shown that PAD amplitude was decreased by $60 \%$ during scratch compared with rest. The last two findings are taken as evidence that the scratching task is associated with a depression of transmission in at least some presynaptic inhibitory pathways. These differences constitute for us clear evidence for the existence of task-specific transmission in the pathways of presynaptic inhibition.

The above results strongly suggest that there is a relationship between the decrease in antidromic firing and the decrease in PAD transmission during scratch. This also implies that PAD pathways are responsible for most of the antidromic firing and that CPG-related oscillations are not. For example, the peak depolarization in dorsal rootlet usually occurs during the flexion phase, whereas rhythmic bursts of antidromic firing can occur anywhere in the step cycle (Dubuc et al., 1985, 1988; Beloozerova and Rossignol, 1994, 1999). However, maximal PAD evoked by sensory inputs can be reached in different phases of the fictive step cycle as recorded in individual muscle group I afferents (Ménard et al., 1999). Also, growing evidence does not support a link between CPG-related oscillations and PAD pathways in the cat. For instance, the phasic pattern of the CPG-related oscillations and the pattern of phase-dependent modulation of PAD (or DRPs) evoked by sensory volleys may be completely different (Gossard and Rossignol, 1990; Gossard et al., 1990). Furthermore, double intracellular recordings have revealed that the CPG-related depolarization in IA axons had no effect on their
A
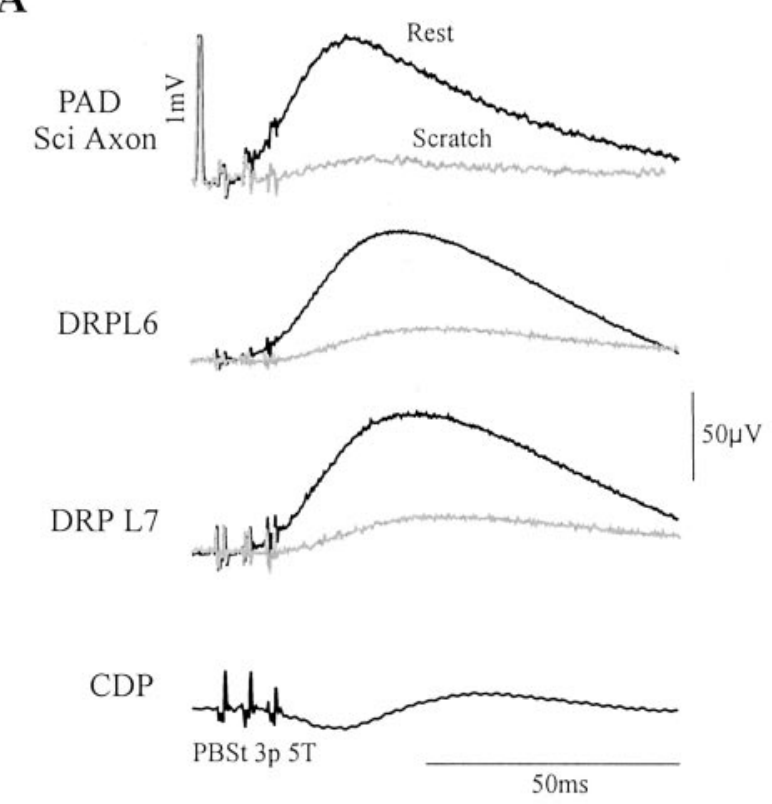

B

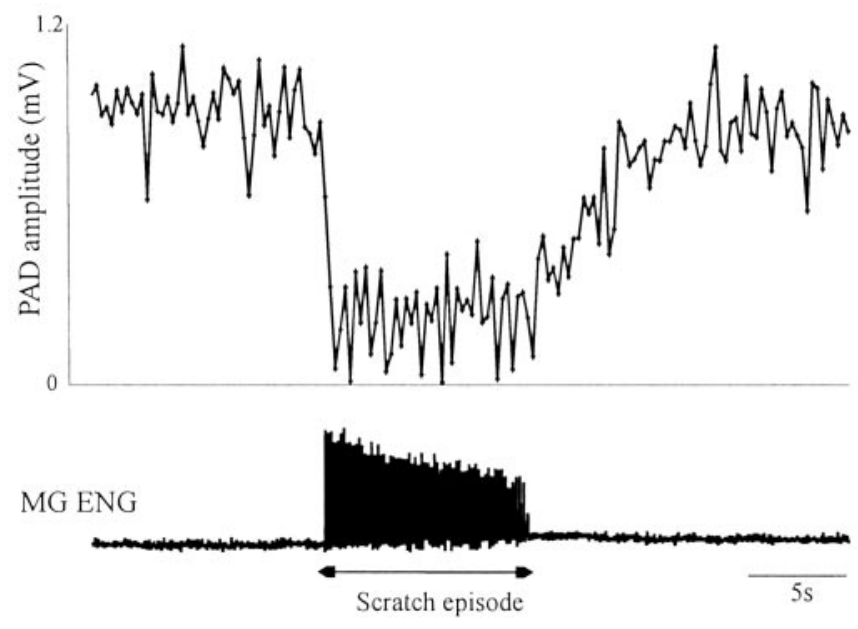

Figure 6. Sensory-evoked PAD and DRP during rest and fictive scratch. A, Top to bottom, Averaged PAD in a sciatic axon, DRP in an L7 rootlet, and DRP in an S1 rootlet evoked by PBSt stimuli at rest (black) and during fictive scratch ( gray) are superimposed. The CDP shows incoming volleys. Calibration: $1 \mathrm{mV}$. B, PADs amplitude evoked repetitively $(3.3 \mathrm{~Hz})$ is linked by a line during an episode of rest and fictive scratch and back to rest. The rectified ENG from MG showing activity during the episode of scratch is at the bottom.

transmission to motoneurons, whereas the sensory-evoked PAD was clearly inhibitory (Gossard, 1996). Likewise, no differences were found in the stretch and H-reflex amplitude in two ankle extensors of decerebrated cats measured for similar EMG levels during tonic contractions and locomotor contractions, confirming that CPG activity on its own does not change transmission from IA axons (Misiaszek et al., 2000). Therefore, we propose that the CPG-related oscillations in afferent potential are not generated through PAD pathways but may instead reflect varying levels of potassium concentrations, as observed previously during fictive locomotion in the cat (Duenas and Rudomin, 1988) and in the neonatal rat (Kremer and Lev-Tov, 1998), as well as during respiration (Richter et al., 1978). 
If the end result of presynaptic activity is to depress the efficacy of sensory transmission (Eccles, 1964; Schmidt, 1971; Nicoll and Alger, 1979; Rossignol et al., 1998; Rudomin and Schmidt, 1999), then our findings suggest that sensory inputs should have a profound influence on spinal cord pathways during scratch. One indication of such influence is the difference between fictive and real scratch movements. During the fictive scratching cycle, as reported here, there is a brisk burst of activity in extensors, whereas the activity in flexors lasts much longer. During the real scratching cycle, when the paw can make contact with the irritated skin, the duration of extensor (medial gastrocnemius) activity is greatly prolonged relative to the activity of flexors (Kuhta and Smith, 1990). Sensory feedback, possibly from force transducers, could be responsible for prolonging the extensor activity (Kuhta and Smith, 1990). A decrease in presynaptic inhibition and antidromic firing as reported here would facilitate such peripheral influence. Our results, based on fictive motor tasks, reveal the influence of central networks on the transmission of presynaptic inhibitory pathways. However, sensory inputs from peripheral receptors stimulated during real movements should also influence transmission in these pathways, and the resulting level of presynaptic inhibition is unknown at present.

Finally, as mentioned before, rhythmic bursts of antidromic firing are commonly found during fictive and real locomotion, whereas they are extremely rare during fictive scratch (Côté and Gossard, 2001). This difference could simply be because of the relatively larger PAD amplitude during locomotion, as reported in this study, reaching more readily firing threshold. As reported previously, such antidromic bursts may exert considerable depression of sensory coding in peripheral receptors (Bévengut et al., 1997; Gossard et al., 1999). One putative role of such peripheral barrage could be to filter predictable from unpredictable sensory inputs (Gossard et al., 1989, 1991; Rossignol, 1996; Nusbaum et al., 1997; Ménard et al., 1999). Indeed, stepping movements should be more exposed to perturbations (e.g., obstacles) than the limited scratch movement. An increased level of presynaptic activity during stepping could be used to focus on unpredictable sensory feedback necessary to adjust and correct the ongoing movement.

\section{References}

Baev KV (1980) Polarization of primary afferent terminals in the lumbar spinal cord during fictitious locomotion. Neurophysiology 12:305-311.

Baev KV, Kostyuk PG (1981) Primary afferent depolarization evoked by the activity of spinal scratching generator. Neuroscience 6:205-215.

Baev KV, Kostyuk PG (1982) Polarization of primary afferent terminals of lumbosacral cord elicited by the activity of spinal locomotor generator. Neuroscience 7:1401-1409.

Beloozerova IN, Rossignol S (1994) Antidromic activity of dorsal root filaments during treadmill locomotion in thalamic cats. Soc Neurosci Abstr 20:1755.

Beloozerova IN, Rossignol S (1999) Antidromic discharges in dorsal roots of decerebrate cats. I. Studies at rest and during fictive locomotion. Brain Res 846:87-105.

Bévengut M, Clarac F, Cattaert D (1997) Antidromic modulation of a proprioceptor sensory discharge in crayfish. J Neurophysiol 78:1180-1183.

Brooke JD, Collins DF, Boucher S, McIlroy WE (1991) Modulation of human short latency reflexes between standing and walking. Brain Res 548:172-178.

Cattaert D, El Manira A, Clarac F (1994) Chloride conductance produces both presynaptic inhibition and antidromic action potentials in primary afferents. Brain Res 666:109-112.

Clarac F, El Manira A, Cattaert D (1992) Presynaptic control as a mechanism of sensory-motor integration. Curr Opin Neurobiol 2:764-769.

Côté M-P, Gossard J-P (2000) Modulation of dorsal root potentials during fictive scratching in the cat. Soc Neurosci Abstr 26:1233.
Côté M-P, Gossard J-P (2001) Task-dependant modulation of presynaptic inhibition in the cat. Canadian Physiological Society, Proceedings of the 2001 Winter Meeting, Mont Tremblant, Canada.

Deliagina TG, Feldman AG, Gelfand IM, Orlovsky GN (1975) On the role of central program and afferent inflow in the control of scratching movements in the cat. Brain Res 100:297-313.

Deliagina TG, Orlovsky GN, Perret C (1981) Efferent activity during fictitious scratch reflex in the cat. J Neurophysiol 45:595-604.

DiCaprio RA (1999) Gating of afferent input by a central pattern generator. J Neurophysiol 81:950-953.

Dietz V, Discher M, Faist M, Trippel M (1990) Amplitude modulation of the human quadriceps tendon jerk reflex during gait. Exp Brain Res $82: 211-213$.

Dubuc R, Cabelguen J-M, Rossignol S (1985) Rhythmic antidromic discharges of single primary afferents recorded in cut dorsal root filaments during locomotion in the cat. Brain Res 359:375-378.

Dubuc R, Cabelguen J-M, Rossignol S (1988) Rhythmic fluctuations of dorsal root potentials and antidromic discharges of primary afferents during fictive locomotion in the cat. J Neurophysiol 60:2014-2036.

Duenas SH, Rudomin P (1988) Excitability changes of ankle extensor group Ia and Ib fibers during fictive locomotion in the cat. Exp Brain Res $70: 15-25$.

Eccles JC (1964) Presynaptic inhibition in the spinal cord. Prog Brain Res 12:65-91.

Eccles JC, Eccles RM, Magni F (1961) Central inhibitory actions attributable to presynaptic depolarization produced by muscle afferent volleys. J Physiol (Lond) 159:147-166.

Faist M, Dietz V, Pierrot-Deseilligny E (1996) Modulation, probably presynaptic in origin, of monosynaptic Ia excitation during human gait. Exp Brain Res 109:441-449.

Feldberg W, Fleischhauer K (1960) Scratching movements evoked by drugs applied to the upper cervical cord. J Physiol (Lond) 151:502-517.

Gossard J-P (1996) The control of transmission in muscle group IA afferents during fictive locomotion in the cat. J Neurophysiol 76:4104-4112.

Gossard J-P, Rossignol S (1990) Phase-dependent modulation of dorsal root potentials evoked by peripheral nerve stimulation during fictive locomotion in the cat. Brain Res 537:1-13.

Gossard J-P, Cabelguen J-M, Rossignol S (1989) Intra-axonal recordings of cutaneous primary afferents during fictive locomotion in the cat. J Neurophysiol 62:1177-1187.

Gossard J-P, Cabelguen J-M, Rossignol S (1990) Phase-dependent modulation of primary afferent depolarization in single cutaneous primary afferents evoked by peripheral stimulation during fictive locomotion in the cat. Brain Res 537:14-23.

Gossard J-P, Cabelguen J-M, Rossignol S (1991) An intracellular study of muscle primary afferents during fictive locomotion in the cat. J Neurophysiol 65:914-926.

Gossard J-P, Bouyer L, Rossignol S (1999) The effects of antidromic discharge on the orthodromic firing of primary afferents in the cat. Brain Res 825:132-145.

Kremer E, Lev-Tov A (1998) GABA-receptor-independent dorsal root afferents depolarization in the neonatal rat spinal cord. J Neurophysiol 79:2581-2592.

Kuhta PC, Smith JL (1990) Scratch responses in normal cats: hindlimb kinematics and muscle synergies. J Neurophysiol 64:1653-1667.

Kurasawa I, Hirose Y, Sunada T, Nakamura Y (1988) Phase-linked modulation of excitability of presynaptic terminals of low-threshold afferent fibers in the inferior alveolar nerve during cortically induced fictive mastication in the guinea pig. Brain Res 446:113-120.

Ménard A, Leblond H, Gossard J-P (1999) The modulation of presynaptic inhibition in single muscle primary afferents during fictive locomotion in the cat. J Neurosci 19:391-400.

Misiaszek JE, DeSerres SJ, Stein RB, Jiang W, Pearson KG (2000) Stretch and $\mathrm{H}$ reflexes in triceps surae are similar during tonic and rhythmic contractions in high decerebrate cats. J Neurophysiol 83:1941-1950.

Morin C, Pierrot-Deseilligny E, Hultborn H (1984) Evidence for presynaptic inhibition of muscle spindle Ia afferents in man. Neurosci Lett 44:137-142.

Nicoll RA, Alger BE (1979) Presynaptic inhibition: transmitter and ionic mechanisms. Int Rev Neurobiol 21:217-258.

Nusbaum MP, El Manira A, Gossard J-P, Rossignol S (1997) Presynaptic mechanisms during rhythmic activity in vertebrates and invertebrates. In: 
Neurons, networks, and motor behavior (Stein PSG, Grillner S, Selverston AI, Stuart DG, eds), pp 237-253. Cambridge, MA: MIT.

Richter DW, Camerer H, Sonnhof U (1978) Changes in extracellular potassium during the spontaneous activity of medullary respiratory neurones. Pflügers Arch 376:139-149.

Richter DW, Jordan D, Ballantyne D, Meesmann M, Spyer KM (1986) Presynaptic depolarization in myelinated vagal afferent fibres terminating in the nucleus of the tractus solitaris in the cat. Pflügers Arch 406:12-19.

Rossignol S (1996) Neural control of stereotypic limb movements. In: Handbook of physiology, Exercise: regulation and integration of multiple systems, Sect 12 (Rudomin P, Romo R, Mendell LM, eds), pp 173-216. Bethesda, MD: American Physiological Society.

Rossignol S, Beloozerova I, Gossard J-P, Dubuc R (1998) Presynaptic mechanisms during locomotion. In: Presynaptic inhibition and neural control mechanisms (Rudomin P, Romo R, Mendell LM, eds), pp 385-397. New York: Oxford UP.

Rudomin P, Schmidt RF (1999) Presynaptic inhibition in the vertebrate spinal cord revisited. Exp Brain Res 129:1-37.

Schmidt RF (1971) Presynaptic inhibition in the vertebrate central nervous system. Ergeb Physiol 63:20-101.

Stein RB, Capaday C (1988) The modulation of human reflexes during functional motor tasks. Trends Neurosci 11:328-332.

Westberg KG, Kolta A, Clavelou P, Sandström G, Lund JP (2000) Evidence for functional compartmentalization of trigeminal muscle spindle afferents during fictive mastication in the rabbit. Eur J Neurosci 12:1145-1154.

Yang JF, Whelan PJ (1993) Neural mechanisms that contribute to cyclical modulation of the soleus H-reflex in walking in humans. Exp Brain Res 95:547-556. 